oscillation of the coupled photon-matter system between its two states, where one of the two systems, material or photon, is in the excited state while the other is in the ground state, and vice-versa.

C. Weisbuch (Ecole Polytechnique, Paris) described the strongly-coupled system realized by inserting semiconductor quantum wells in a planar Fabry-Perot microcavity consisting of monolithically-integrated, directly-grown, quarter-wave layer stacks of mirrors (a so-called distributed-Bragg reflector or DBR) sandwiching a spacer layer (Fig. 2a). The most recent work [3] deals with the observation and understanding of coupledmode luminescence. The in-plane momentum dispersion curves of cavity polaritons (the coupled-mode dispersion) has been determined from a detailed analysis of the angular-resolved emission and is found to agree with the predicted curves (Fig. 2b). One therefore now understands the basic aspects of cavity-polariton emission.

There remain, however, some aspects that need further work. In particular Y. Yamamoto (Stanford and Tokyo) discussed the effects observed at high intensity with stronglycoupled semiconductor quantum wells in microcavities. He modified the usual lightmatter Hamiltonian describing the photon field, the material system, and their interaction by inserting boson operators. The solution led to sidebands instead of the usual Rabi doublet, However, this attributing of luminescence sidebands to "Rabi sidebands" originating in the boson nature of the excitons is at variance with the normal fermionic behaviour of electrons in atoms.

Besides the special session, several presentations also dealt with photon-controlled structures. F. Yang et al. (Cardiff) reported a $25 \%$ improvement of the threshold of horizontal Fabry-Perot lasers using a planar microcavity to suppress unwanted spontaneous emission into vertical modes. Stanley et al. (EPF-Lausanne) demonstrated stronglycoupled multiple microcavities, whereby one couples a material system (quantum wells) with several photon-coupled microcavities. Being able to independently specify the coupling strength between the various oscillators gives a welcome handle on the concept of variable or heterogeneous coupling (the cavities, for instance, could associate different materials and functionalities)

\section{High Efficiency LEDs}

But the major issue in the field is to improve light-emitting devices (LEDs). Research is active world-wide and could soon provide a major breakthrough in the high-efficiency generation of light. One of the most desirable devices is the "zero-threshold" laser, where suppression of spontaneous emission in all photon modes but one, i.e. the laser mode itself, allows continuous switching from spontaneous to stimulated emission (photon number in the remaining mode $>1$ ), with a constant quantum efficiency near unity. Additionally, the emission process would become fully deterministic, which could lead to photon emission that is limited by Johnson noise instead of the much stronger shot noise arising from the coexistence of random spontaneous emission with laser emission. De Neve et al. (IMEC, Gent) reported an important step towards the realization of high-efficiency LEDs, possibly
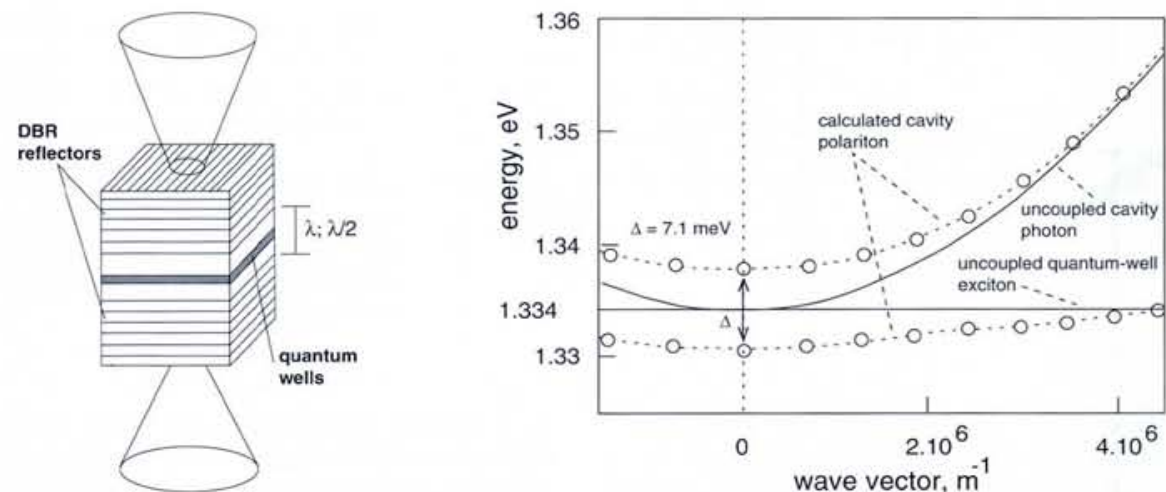

Fig. 2 - Polaritons in a planar semiconductor microcavity. a, left) Semiconductor quantum wells are inserted in a planar Fabry-Perot microcavity consisting of monolithically-integrated, directly-grown, quarter-wave layer stacks of mirrors (a so-called distributed-Bragg reflector or $D B R$ ) sandwiching a spacer layer with a thickness of $\lambda / 2$ or $\lambda$. Strongly coupled behaviou arises when the cavity resonant photon mode parallel to the layers resonates with the quantum-well exciton. $b$, right) In-plane dispersion of the coupled exciton-cavity photon mode as determined from angular-resolved luminescence measurements. The experimental data points are accurately described by the coupled-mode dispersion (lines), the so-called cavity polariton, calculated by assuming that the excitons and the resonant cavity form a simple system of two oscillators coupled through the light-matter interaction.

zero-threshold lasers, by carefully designing a planar microcavity to give an external quantum efficiency of $6.2 \%$ (recently extended to $10.5 \%$ ).

[1] Yablonovitch E., J. Opt. Soc. Amer. B 10

\section{Austrian Textbook Wins Amaldi Prize}

Reconciling our everyday world with the complex logical construction based on empirical principles and mathematics that allows natural phenomena to be modelled is seen by many as the challenge facing secondary school teachers. The winner of the 1993 Amaldi International Prize for a High-School Physics textbook addresses this problem by splitting the physics course into its essential parts (as four, short compact, volumes on basic knowledge) complemented by series of 12 theme-oriented books dealing with applications in daily life. The books also introduce some novel but useful devices, such as logos asking the student to do something (e.g., "think about ..."), ideas for experiments for the at home, and English translations of technical terms.

The winning textbook is called Physikcompact published by Verlag Hölder-PilcherTempsky (Vienna), Austria's largest publisher of maths and physics textbooks. The authors are Albert Jaros, Alfred Nussbaumer, Peter Nussbaumer, Hansjoerg Kunze, Leopold Mathelitsch, Robert Hofstetter, and Hans Haimo Tentchert. R. Hofstetter teaches physics and philosophy at Vienna University and in a gymnasium and L. Mathelitsch is Professor of Theoretical Physics at the Technical University, Graz. The other authors are all teachers in gymnasia, with the two Nussbaumers being twin brothers.

Physik-compact was published to coincide with the introduction of Austria's new physics curriculum in 1989, which the authors were heavily involved in defining. The four basic volumes (Baiswissen) are only sold in Austria, but some 1500 of the theme-oriented books (Themenhefte) have been sold in Germany. The Prize Jury felt the contents were flexible enough to suit curicula in different countries. This may encourage the publication of translations, for which there are no plans at present.
(1993) 283; J. Mod. Optics 41 (1994) 171

[2] See, e.g., Slusher R.E. \& Weisbuch C. Solid State Commun. 92 (1994) 149.

[3] Houdre R. et al., Phys. Rev. Lett. 73 (1994) 2043

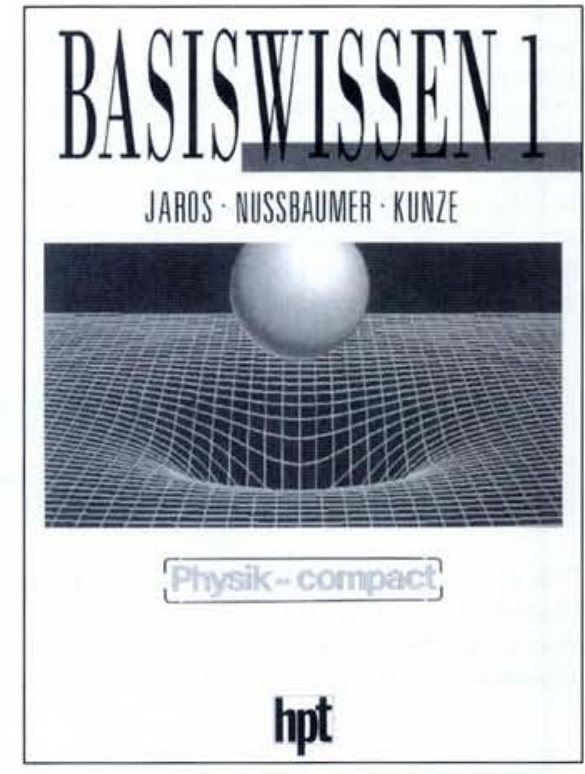

Physik-compact

4 main books ( 452 p.): AS515.-

12 theme-oriented books (464p.): AS 597.Publisher: Verlag Hölder-Pichler-Tempsky Jochen-Rindt-Strasse 11, A-1232 Vienna Tel.: +43-1-4389930

The 1993 Amaldi Prize, the first ever, was awarded by the Edoardo Amaldi Foundation, Piacenza, with EPS sponsorship. It comprised 20000 ECU given to the authors and publishers of a high-school physics textbook first printed in Europe between 1 January 1987 and 30 September 1993. A total of 47 books were submitted out of an estimated $60-70$ potential candidates, there being none in French and relatively few in German. The next prize will be awarded in a few years time. 